\title{
Percepción de los niveles personal, familiar, educativo, social y afectivo en internos de la penitenciaria de Acacias, Meta
}

\author{
Fernanda Ballen ${ }^{1}$ \\ Ana María Reyes ${ }^{1}$ \\ Francy Rodríguez ${ }^{1}$
}

Recibido: 03-03-2017

Aceptado: 11-05-2017

\section{RESUMEN}

En este artículo se presentan los resultados de la investigación realizada en la penitenciaría de Acacías - Meta, con la cual se pretendió conocer, identificar y describir la percepción de los internos del pabellón 4 de dicho penal, sobre los niveles personal, familiar, educativo, social y afectivo de acuerdo a los sistemas del modelo ecológico.

Para comenzar, la teoría del modelo ecológico postulada por Brofenbrenner, consiste en el estudio científico de la progresiva acomodación mutua, entre un ser humano activo en desarrollo y las propiedades cambiantes de los entornos inmediatos en los que vive la persona (sistemas) (1987, p. 40), lo cual es la "interrelación de diferentes elementos ya sean objetos, situaciones o elementos, procesos, variables, de manera organizada los cuales siempre tienen determinada función" (p. 7).

De esta manera, la situación de encarcelamiento es una experiencia difícil de asimilar para la persona privada de la libertad y sus familias, al menos en los primeros momentos del ingreso en prisión, la cual genera en estas personas un esfuerzo adaptativo constante que, como consecuencia, le genera una serie de distorsiones afectivas, emocionales, cognitivas y perceptivas (Altamirano, 2013), puesto que existen restricciones, como el aislamiento emocional y la consecuente frustración (Arroyo, 2011); así mismo existen factores que impactan en el individuo, y los cuales se encuentran asociados a la misma condición de internamiento, 
como la privación de libertad, el alejamiento de las redes sociales y familiares, el abandono de la vida laboral, estrés cognitivo asociado a las preocupaciones por la situación de la familia, el hacinamiento, la falta de privacidad, entre otros (Ruiz, 1999).

Se tiene por objetivo general conocer la percepción de los internos del pabellón 4 de la penitenciaria de Acacias- Meta sobre los niveles de los sistemas del modelo ecológico (familiar, educativo, social y afectivo).

En cuanto a la metodología realizada fue de enfoque cualitativo, diseño fenomenológico, una muestra de 22 internos del pabellón 4 entre las edades de 19 a 44 años; para la recolección de datos se aplicó una entrevista a profundidad. Por último, se realizó el análisis de información por medio del programa Atlas Ti, el cual es una herramienta flexible que permite analizar varias vertientes y por lo tanto facilita en gran parte la organización, manejo e interpretación de datos cualitativos (Cuevas y Méndez, 2012).

Palabras clave: modelo ecológico, penitenciaria, percepción, encarcelamiento, internos.

\title{
Perception of the personal, family, educational, social and emotional levels of the Acacias penitentiary, Meta
}

\begin{abstract}
In this article presents the results of research based on understanding This study aimed to identify, identify and describe the perception of the inmates of Hall 4 of the Acacias-Meta penitentiary on personal, family, educational, social and affective levels according to the ecological model.

To begin with, the theory of the ecological model postulated by Brofenbrenner (1987) consists in the scientific study of the progressive mutual accommodation between an active human being in development and the changing properties of the immediate environments in which the
\end{abstract}


person lives (systems) ( .40 ), which is the "interrelationship of different elements, whether objects, situations or elements, processes, variables, in an organized way which always have a certain function" (p.7).

In this way, the situation of incarceration is an experience difficult to assimilate for the person deprived of liberty and their families, at least in the first moments of prison, which generates in these people a constant adaptive effort that, as a consequence, Generates a series of affective, emotional, cognitive and perceptive distortions (Altimirano, 2013), since there are restrictions such as emotional isolation and frustration (Arroyo, 2011); There are also factors that impact on the individual, and which are associated with the same condition of internment, such as deprivation of freedom, withdrawal from social and family networks, withdrawal from working life, cognitive stress associated with concerns By family situation, overcrowding, lack of privacy, among others (Ruiz, 1999).

It has as a general objective To know the perception of the inmates of Hall 4 of the penitentiary of Acacias-Meta on the levels of the systems of the ecological model (family, educational, social and affective).

As for the methodology was a qualitative approach, phenomenological design, a sample of 22 inmates of ward 4 between the ages of 19 to 44 years; For data collection an in-depth interview was applied. Finally, the analysis of information was carried out through the Atlas Ti program, which is a flexible tool that allows analyzing several aspects and therefore greatly facilitates the organization, management and interpretation of qualitative data (Cuevas and Méndez, 2012).

Keywords: Ecological model, penitentiary, perception, imprisonment, inmates.

\section{Introducción}

En el presente artículo se muestra el trabajo de investigación, a través del cual se pretendió conocer, identificar y describir la percepción de los internos del pabellón 4 de la penitenciaria de Acacias- Meta sobre los niveles personal, familiar, educativo, social y afectivo de acuerdo a los sistemas del modelo ecológico. 
Para comenzar, la teoría del modelo ecológico postulada por Brofenbrenner, consiste en el estudio científico de la progresiva acomodación mutua entre un ser humano activo en desarrollo y las propiedades cambiantes de los entornos inmediatos en los que vive la persona (sistemas) $(1987$, p. 40), lo cuál es la "interrelación de diferentes elementos ya sean objetos, situaciones o elementos, procesos, variables, de manera organizada los cuales siempre tienen determinada función" (p. 7).

Según la teoría del modelo ecológico los sistemas en los que se desarrolla el ser humano son los siguientes:

- (microsistema) nivel familiar y personal relacionado con

autoestima, auto concepto y autoimagen.

- (mesosistema) nivel afectivo y social relacionado con la dinámica familiar y afectiva.

- (exosistema) nivel educativo relacionado con productividad, desempeño y satisfacción a modo de aprendizaje educativo.

- (macrosistema) el entorno (la penitenciaria) relacionado con relaciones interpersonales.

Por otra parte, con relación a la percepción, según Vargas (1994), es el proceso mediante el cual depende la ordenación, clasificación y elaboración de sistemas de categorías con los que se comparan los estímulos que el sujeto recibe, ya que conforma los referentes perceptuales a través de los cuales se identifican las nuevas experiencias sensoriales transformándolas en eventos reconocibles y comprensibles dentro de la concepción colectiva de la realidad (p. 47).

Teniendo en cuenta lo anterior, según Altamirano (2013), el suceso de encarcelamiento es uno de los acontecimientos más traumáticos que puede vivenciar una persona, debido al aislamiento social y familiar al que está expuesto, así mismo, el esfuerzo adaptativo al entorno penitenciario genera una serie de distorsiones emocionales, psicológicas y físicas. De igual modo, a lo largo del proceso de investigación se evidencian mayores reacciones y consecuencias que puede generar el evento de prisionialización.

En cuanto a la metodología realizada fue de enfoque cualitativo, diseño fenomenológico; se tomó una muestra de 22 internos del pabellón 4 entre las edades de 19 a 44 años; para la recolección de datos 
se aplicó una entrevista a profundidad. Por último, se realizó el análisis de información por medio del programa Atlas Ti, el cual es una herramienta flexible que permite analizar varias vertientes y por lo tanto facilita en gran parte la organización, manejo e interpretación de datos cualitativos (Cuevas y Méndez, 2012).

Basados en los resultados del presente estudio, sobre la percepción de los niveles del modelo ecológico, se pudieron evidenciar las siguientes categorías, subcategorías y categorías emergentes:

-Categorías: nivel personal, nivel familiar, nivel social, nivel educativo y nivel afectivo.

-Subcategorías: desmotivación, tristeza, baja autoestima y espiritualidad.

-Categorías emergentes: insatisfacción personal, resiliencia, frustración, conductas inadecuadas, habilidades sociales, soledad, red de apoyo, relaciones interpersonales, resocialización, autopercepción, autorrealización y resentimiento.

Por último, se concluye que existe una relación entre la percepción que tienen los internos en cuanto a los niveles (personal, familiar, educativo, social y afectivo), del modelo ecológico y así mismo tienen un impacto sobre la persona y su desarrollo psicológico teniendo en cuenta las condiciones de encarcelamiento.

Así mismo, el modelo propuesto ha demostrado un potencial para avanzar en la comprensión del fenómeno, considerando las características complejas, y así mismo ofrece una comprensión de los ambientes inmediatos en el que la persona se desarrolla, permitiendo focalizar los puntos de dificultades y/o favorecimiento y dando la posibilidad de generar nuevas estrategias para la prevención de problemáticas en los diferentes niveles del sistema ecológico de las personas.

\section{Percepción de los internos desde el modelo ecológico}

Marco contextual: la investigación fue realizada en el Establecimiento Penitenciario y Carcelario de Mediana Seguridad de Acacias- Meta, el 
cual se encuentra ubicado a cinco kilómetros del perímetro urbano del municipio de Acacias; esta penitenciaria tiene una capacidad de 2.376 internos y cuenta con 2.594 hasta el 2015 (Inpec, 2015).

Actualmente, en el patio 4 de la Penitenciaria de Mediana Seguridad Carcelaria de Acacias, existen 198 internos hombres, que hacen presencia debido a una variedad de delitos, por lo cual deben cumplir una sentencia o pena legal, acorde con la normatividad relacionada con el delito incurrido, sin embargo; en este ambiente se evidencian situaciones que son reflejo de un liderazgo inadecuado y conflictivo, ya que pertenecen a distintos grupos al margen de la ley, y así mismo de diferentes creencias y formas de pensar, ocasionando división por grupos en el mismo patio. Basándose en lo anterior, se están generando grupos aislados, comportamientos inadecuados de disociación tanto individual como grupal, eventos que pueden generar actos de violencia, conflicto, división del grupo; ocasionando una problemática para el establecimiento y de convivencia dentro del patio. Por las consideraciones anteriores, se observa claramente que factores como la falta de sensibilización, respeto hacia sus compañeros, falta de comunicación asertiva y otros generan una problemática de convivencia (Suarez \& Ávila, 2014).

Marco conceptual: es fundamental mencionar el concepto de percepción, ya que los internos al estar en condición de encarcelamiento experimentan una serie de cambios en cuanto a su percepción y cognición a nivel personal, familiar, educativo, social y afectivo en el que se desarrollaron. Por ende, según Rivera, Arellano y Molero (2000) definen este término como: "conjunto de procesos y actividades relacionados con la estimulación que alcanza los sentidos, mediante los cuales obtenemos información respecto a nuestro hábitat, las acciones que efectuamos en él y nuestros propios estados internos" (p. 68).

Así mismo, cabe mencionar y relacionar el presente trabajo de investigación con el área de psicología social, entendiéndose este término como el estudio científico de los efectos de los procesos sociales y cognitivos sobre el modo en que los individuos perciben, influyen, se relacionan, comprenden e interactúan unos con otros (Eliot y Mackie, 1997).

Marco teórico: la investigación está referida teóricamente desde el modelo ecológico postulado por Bronfenbrenner en 1987, el cual defiende una visión integral, sistémica y naturalista del desarrollo 
psicológico entendido como un proceso complejo, que responde a la influencia de una multiplicidad de factores estrechamente ligados al ambiente o entorno ecológico en el que dicho desarrollo tiene lugar (Brofenbrenner, 1987).

Microsistema: corresponde al patrón de actividades, roles y relaciones interpersonales que la persona en desarrollo experimenta en un entorno determinado en el que participa (Brofenbrenner, 1987). Por ejemplo, se sitúan los principales contextos socializadores, es decir, la familia, la pareja, los pares, amigos, entre otros entornos cercanos que permiten una interacción dinámica. Por otro lado, se considera que si la dinámica de roles entra en conflicto la persona experimentaría un dilema constante por tratar de cubrir de forma simultanea todas sus obligaciones lo cual puede generar una disonancia comportamental debido por sentimientos de frustración, ansiedad y angustia (Cohen, 1973).

Mesosistema: comprende las interrelaciones de dos o más entornos (microsistemas) en los que la persona en desarrollo participa (por ejemplo, para un niño, las relaciones entre el hogar, la escuela y el grupo de pares del barrio; para un adulto, entre la familia, el trabajo y la vida social) (Brofenbrenner, 1987). Es decir, una persona amplía su mesosistema a medida que se originan nuevas necesidad fisiológicas y sociológicas que cumplir, lo cual va generando la incorporación o integración de nuevos entornos al sistema (Pérez, Guerra, Gutiérrez y Villafranca, 2011).

Exosistema: se refiere a los propios entornos (uno o más), en los que la persona en desarrollo no está incluida directamente, pero en los que se producen hechos que afectan a lo que ocurre en los entornos en los que la persona si está incluida (para el niño, podría ser el lugar de trabajo de los padres, la clase del hermano mayor, el círculo de amigos de los padres, las propuestas del Consejo Escolar, etc.) (Brofenbrenner, 1987).

Macrosistema: se refiere a los marcos culturales o ideológicos que afectan o pueden afectar transversalmente a los sistemas de menor orden (micro-, meso- y exo-) y que les confiere a estos una cierta uniformidad, en forma y contenido, y a la vez una cierta diferencia con respecto a otros entornos influidos por otros marcos culturales o ideológicos diferentes (Brofenbrenner, 1987). 
Por último, el macrosistema contiene todos los sistemas que organizan la dinámica de una sociedad, es decir, las leyes y normas establecidas por el Estado, lineamientos culturales que molden el comportamiento de las personas entre otros elementos (Brofenbrenner, 1987).

Como resultado se obtuvo que en el microsistema, en la categoría de nivel familiar no se evidenciaron subcategorías, teniendo en cuenta que los internos solo mostraron interés por el apoyo negativo o positivo por parte de sus familias, especialmente de la madre; en las categorías emergentes se halló la red de apoyo y la soledad concebida como un estado subjetivo que se contrasta con la condición de aislamiento físico, la cual surge como una respuesta ante la falta de una relación particular (Montero, López y Sánchez, 2001); en el mesosistema, con respecto a la categoría a nivel afectivo, se encontró la subcategoría de decepción puesto que mencionan haber defraudado a las personas que los rodean he incluso a ellos mismos. Dentro de las categorías emergentes de este nivel, se encontró nuevamente la soledad, además del resentimiento, especialmente hacia aquellas personas que los han juzgado por su condición de internos y a su vez los han abandonado; así mismo en la categoría a nivel social, la cual está conformada por los entornos inmediatos donde la persona interactúa; se encontró la subcategoría de desadaptación al contexto, puesto que a la mayoría de los participantes se les ha dificultado adaptarse y/o cumplir con las normas establecidas por el Inpec; por el contrario en las categorías emergentes se evidenciaron las relaciones interpersonales, (amigos, buenos compañeros, entre otros conceptos dado por los internos), la resocialización, (participan en programas de atención y tratamiento), la autopercepción y la red de apoyo. En el exosistema, con respecto a lo hallado en las subcategorías a nivel educativo, se encontraron las metas, donde cada uno de los internos resalta que aun estando en condiciones de encarcelamiento desean salir adelante, e incluso lograr ser profesionales. Según las categorías emergentes, se encontró la autorrealización, y finalmente en el macrosistema, el cual contiene todos los sistemas que organizan la dinámica de una sociedad, es decir, las leyes y normas establecidas por el Estado, lineamientos culturales que moldean el comportamiento de las personas entre otras (Brofenbrenner, 1987). Partiendo de lo anterior el macrosistema es el Establecimiento Penitenciario de Mediana Seguridad y Carcelario de Acacias Meta, y el pabellón 4 del mismo establecimiento, es el entorno y la realidad donde conviven y se desenvuelven diariamente cada uno de los participantes. 


\section{Conclusiones}

A partir del estudio del modelo ecológico en los internos del Establecimiento Penitenciario y Carcelario de Acacias- Meta, pueden establecerse las siguientes conclusiones:

En la muestra de la presente investigación se encuentran 22 internos de sexo masculino, que oscilan entre las edades de 19 y 44 años, con estado civil prevalente soltero, nivel de escolaridad desde sexto de bachillerato hasta nivel técnico, y finalmente en situación jurídica se encuentran internos por los delitos de hurto, tráfico de estupefacientes y homicidio.

En cuanto al modelo ecológico, se logró identificar en el microsistema (familiar y personal), que los internos perciben tener una red de apoyo familiar, pero así mismo sienten desmotivación debido al poco tiempo que comparten con sus familiares, baja autoestima, sentimientos de tristeza, frustración y mala conducta que surge por las condiciones de encarcelamiento.

En el mesosistema (afectivo y social), se encontró que los internos perciben en el nivel afectivo decepción, soledad y resentimiento, debido al abandono de sus parejas, esposas y amigos, generado posteriormente al evento de encarcelamiento. Por otro lado, a nivel social, la mayoría de los internos perciben desadaptación al contexto, debido a la cultura del pabellón y encuentran una red de apoyo generada por sus mismos compañeros de patio (pares).

En el exosistema (nivel educativo), se evidenció que los internos perciben tener establecidas metas, las cuales ayudan al proceso de autorrealización en torno a las oportunidades educativas que les brinda el Inpec (talleres de emprendimiento, actividades culturales, estudios de primaria y media básica, entre otras), las cuales aportan a la resocialización.

Por último, en el macrosistema (contexto penitenciario), se logró identificar que los internos perciben el contexto penitenciario como un lugar en el cual se les brinda varias oportunidades de aprendizaje en diferentes áreas, por lo tanto, se sienten conformes con la instancia en este contexto, teniendo en cuenta las leyes y normas por las cuales se rige la penitenciaria. 
Partiendo de lo anterior, en el presente estudio se pudo evidenciar que existe una relación entre la percepción que tienen los internos, en cuanto a los niveles (personal, familiar, educativo, social y afectivo) del modelo ecológico, y así mismo tienen un cambio de manera personal y su desarrollo psicológico, teniendo en cuenta las condiciones de encarcelamiento.

El modelo propuesto ha demostrado un potencial para avanzar en la comprensión del fenómeno, considerando las características complejas, y así mismo, ofrece una comprensión de los ambientes inmediatos en el que la persona se desarrolla, permitiendo focalizar los puntos de dificultades, favorecimiento y dando la posibilidad de generar nuevas estrategias para la prevención de problemáticas en los diferentes niveles del sistema ecológico de las personas.

De acuerdo a los resultados encontrados en el presente estudio, se recomienda al Establecimiento Penitenciario y Carcelario de Mediana Seguridad de Acacias- Meta, empezar a generar mayores programas de atención y prevención teniendo en cuenta el modelo ecológico de los internos, para generar bienestar en cada uno de los ámbitos, puesto que debido a la condición de encarcelamiento generan distintas reacciones a nivel microsistema, mesosistema, exosistema y macrosistema.

Por otro lado, se recomienda a la población carcelaria y penitenciaria la participación en los diversos programas que brinda el Inpec, los cuales están dirigidos en torno a la resocialización, lo que genera en ellos oportunidades laborales, económicas y educativas. Adicionalmente, estas actividades/programas ayudan al desarrollo y fortalecimiento en las relaciones personales e interpersonales, teniendo en cuenta los niveles del modelo ecológico.

Por último, para futuras investigaciones, se recomienda realizar estudios desde la perspectiva del modelo ecológico, puesto que permite estudiar los ambientes en lo que se desarrolla el ser humano, y por ende la influencia que estos sistemas (micro, meso, exo y macrosistema) pueden ejercer en él. 


\section{Referencias bibliográficas}

Altamirano, Z. (2013). El bienestar psicológico en prisión: antecedentes y consecuencias. Tesis doctoral, Madrid: Universidad Autónoma de Madrid. Disponible en: http://www. pensamientopenal.com.ar/system/files/2015/01/doctrina40538.pdf

Arroyo, J. (2011). Health care strategies for mental health problems in the prison environment, the Spanish case in a European context. Revista Española de Sanidad Penitenciaria, 13(3), 100-110. Disponible en: http://www.sanipe.es/OJS/index.php/ RESP/article/view/13/58

Bronfenbrenner, U. (1987). La ecología del desarrollo humano. Barcelona: Paidós.

Cohen, J. (1973). Psicodinámica de la Personalidad. México: Trillas.

Cuevas, A., Méndez, S. \& Hernández, R. (2012). Manual de introducción al Atlas ti. Disponible en: : http://metodologia2012.bligoo.cl/media/users/23/1179686/ files/332545/ATLAS.ti_MI5aCD.pdf

Eliot, S. \& Mackie, D. (1997). Psicología social. Madrid: Medical Panamericana.

Instituto Nacional Penitenciario y Carcelario. (2016). Glosario de términos estandarizados del sistema penitenciario y carcelario: general y de Colombia. Disponible en: http://www.inpec.gov.co/portal/page/portal/Inpec/ ServiciosDelnformacionAlCiudadano/Glosario

Instituto Nacional Penitenciario y Carcelario. (2016). Informe estadístico 2016. Disponible en: http://www.inpec.gov.co/portal/page/portal/Inpec/Institucion/ Estad\%EDsticas/Estadisticas/Informes\%20y\%20Boletines\%20Estad\%EDsticos/01\%20 INFORME\%20ENERO\%202016_.pdf

Pérez, J., Guerra, R., Gutiérrez, J. \& Villafranca, M. (2011). Comportamiento del entorno social respecto al embarazo no planeado de jóvenes universitarias: estudio de cuatro casos. Disponible en: http://ftsydh.uanl.mx/wp-content/blogs.dir/6/ejemplaresrealidades/A1-N1.pdf

Rivera, J., Arellano, R. \& Molero, V. (2000). Conducta del consumidor: estrategias y tácticas aplicadas al marketing. Madrid: Esic Editorial. Disponible en: https://books. google.com.co/books?id=7PHK3WfpWPEC\&printsec=frontcover\&hl=es\#v=onepage \&q $\& \mathrm{f}=\mathrm{false}$

Ruiz, J. (1999). Estrés en prisión y factores psicosociales. Revista Colombiana de psicología, (8)0, 120-130. Disponible en: http://www.revistas.unal.edu.co/index.php/ psicologia/article/view/16097/16986 
Suarez, S. y Ávila, J. (2014). El coaching como estrategia de mejoramiento de liderazgo transformacional para el manejo de la problemática de convivencia del patio 4 de la penitenciaria de mediana seguridad y carcelaria de Acacias. Villavicencio Meta: Universidad Cooperativa de Colombia.

Vargas, L. (1994). Sobre el concepto de percepción. Disponible en: http://www.redalyc. org/pdf/747/74711353004.pdf 